\title{
Light and Redox Switchable Molecular Components for Molecular Electronics
}

\author{
Wesley R. Browne* and Ben L. Feringa*
}

\begin{abstract}
The field of molecular and organic electronics has seen rapid progress in recent years, developing from concept and design to actual demonstration devices in which both single molecules and self-assembled monolayers are employed as light-responsive components. Research in this field has seen numerous unexpected challenges that have slowed progress and the initial promise of complex molecular-based computers has not yet been realised. Primarily this has been due to the realisation at an early stage that molecular-based nano-electronics brings with it the interface between the hard (semiconductor) and soft (molecular) worlds and the challenges which accompany working in such an environment. ${ }^{[1-3]}$ Issues such as addressability, cross-talk, molecular stability and perturbation of molecular properties (e.g. inhibition of photochemistry) have nevertheless driven development in molecular design and synthesis as well as our ability to interface molecular components with bulk metal contacts to a very high level of sophistication. Numerous groups have played key roles in progressing this field not least teams such as those led by Whitesides, ${ }^{[1]}$ Aviram, Ratner, ${ }^{[4]}$ Stoddart and Heath. ${ }^{[5]}$ In this short review we will however focus on the contributions from our own group and those of our collaborators, ${ }^{[6]}$ in employing diarylethene ${ }^{[7]}$ based molecular components.
\end{abstract}

Keywords: Diarylethene $\cdot$ Molecular electronics · Self-assembled monolayers

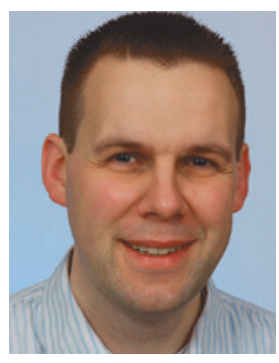

Wesley R. Browne was awarded a BSc in Applied Chemistry in 1999 followed by a $\mathrm{PhD}$ in Chemistry (2002) under the guidance of Prof $\mathrm{J}$. G. Vos at Dublin City University, Ireland on the preparation of isotopically labeled polypyridyl ligands and their use in photophysical studies of $\mathrm{Ru}(\mathrm{II})$ and Os(II) polylpyridyl complexes. This was followed by a postdoctoral position under the joint guidance of Prof $\mathrm{J}$. G. Vos and Prof J. J. McGarvey (Queen's

\footnotetext{
${ }^{\star}$ Correspondence: Dr. W. R. Browne,

Prof. Dr. B. L. Feringa

Centre for Systems Chemistry

Stratingh Institute for Chemistry \& Zernike Institute for Advanced Materials

University of Groningen

Nijenborgh 4, 9747 AG

Groningen, The Netherlands

Fax: +31503634296

Tel.: +31503634235.

E-mail: w.r.browne@rug.nl, b.l.feringa@rug.nl
}

University Belfast, Northern Ireland) on resonance Raman spectroscopy. In 2003 he joined the group of Prof B. L. Feringa as a postdoctoral research. In 2007, he was awarded a VIDI fellowship by the Netherlands Foundation for Science and was appointed Assistant Professor at the University of Groningen in 2008. His current research interests include oxidation catalysis, electro- and photo-responsive molecularly and polymer modified surfaces and Raman spectroscopy.

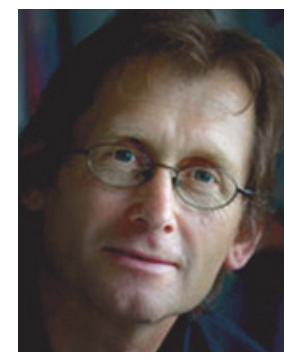

Ben L. Feringa obtained his $\mathrm{PhD}$ in 1978 at the University of Groningen under the guidance of Professor Hans Wynberg. After working as a research scientist at Shell in the Netherlands and the UK, he was appointed lecturer and in 1988 full professor at the University of Groningen and named the distinguished Jacobus $H$. van 't Hoff Professor of Molecular Sciences in 2004. He was elected foreign honorary member of the American Academy of Arts and Sciences and a member of the Royal Netherlands Academy of Sciences. In 2008 he was appointed Academy Professor and was knighted by Her Majesty the Queen of the Netherlands. His research interests range from stereochemistry, organic synthesis, asymmetric catalysis, molecular switches and motors, self-assembly and molecular nano-systems.

\section{Introduction}

Molecular design, synthesis and characterisation of potential functional molecular components for application in molecular and organic electronics is an iterative process in which even very well known compounds can surprise us by their versatility. It is the combination of theory, synthesis and characterisation of light and redox responsive molecules required in this research program that drives curiosity in our research team. Over the last decades we, together with many groups around the world, have been captivated by the remarkable properties demonstrated by a relatively small yet diverse group of responsive molecular systems. As fascinating as these systems are in solution, it is at interfaces between non-molecular materials and on surfaces that attention has shifted to in recent years since application in molecular and organic electronic devices makes this next step inevitable. In this short review, we give a cursory overview of recent advances made by our research teams together with other groups from biochemistry to materials physics. ${ }^{[6]}$

\section{Design Rules for Light-controlled Molecular-based Electronic Components}

At the heart of our research program is the development of light and redox switchable molecules in which at least two stable and often several addition meta-stable 


\section{$\mathrm{A} \underset{\lambda_{2}}{\stackrel{\lambda_{1}}{\rightleftarrows}} \mathrm{B}$}

Scheme 1. Bistable optical molecular switches, where the switching direction is wavelength dependent and photoswitching can be achieved in a fully reversible manner, are ideal candidates as components in molecular electronic devices.

states can be accessed in a fully controlled and reversible manner.

Molecular based photochromic switches can be 'switched' by irradiation with UV and/or visible light between two or more molecular states (A and $\mathbf{B})$ in a reversible manner. The change in molecular structure induced is accompanied by a change in the energies of the frontier molecular orbitals and hence redox and electronic absorption spectra (Scheme 1). ${ }^{[7]}$ It is this change that can be induced by external stimuli that makes these systems attractive as components in molecular-based electronic devices. ${ }^{[8]}$ Furthermore the ability to tune these photochromic systems by synthetic modification together with their often high stability, reversibility and fast response times (i.e. high photochemical quantum yields) provides for a level of control that is unmatched in wholly non-molecular systems. ${ }^{[7]}$

The change in the energy of frontier orbitals that accompanies photoswitching offers the possibility of such photoswitches changing their conductivity in devices, thereby acting, for example, as memory units. ${ }^{[9,10]}$ These photochromic systems, although highly promising, pose major technical challenges to their application in molecular electronics, not least in connecting them to conducting interfaces and in demonstrating that their photochromic state can control conductivity and charge transport significantly. These challenges are compounded by issues such as fatigue, photochemical quantum yields, readout and, critically, on defects. A further complication that is often overlooked is that when confined in a densely packed monolayer these systems can show intermolecular interactions that are not normally observed in solution. [11,12]

\section{Photoswitching of Conductance}

Achieving the full potential of photoswitchable molecular-based electronic devices necessitates that we can control charge transport across the electrode-electrode gap bridged by these systems. This requires that direct tunnelling currents are low relative to mediated charge transport (resonant tunnelling) 'through' molecular orbitals, ${ }^{[13,14]}$ and that the mediated charge transport is significantly different between the two photochromic states. ${ }^{[15]}$ Our own efforts in photo-switching of conductance has focused on the dithienylethene class of photochromes. Dithienylethenes exist in two stable forms; a colourless (open) isomer and a coloured (closed) isomer (Fig. 1). ${ }^{[7]}$ In addition to differences in the electronic absorption spectra of the two isomers between the two forms, there are pronounced differences in redox chemistry and hence in the relative energy levels of the molecular orbitals. ${ }^{[16]}$ It is these changes in frontier orbital energies induced photochemically that we have focused our attention on in developing molecular-based electronic devices in recent years.

\section{Light Switching of Molecules on Metallic Surfaces}

Trans-cis isomerization of azobenzenes, ${ }^{[17-19]}$ stilbenes $^{[20]}$ and overcrowded alkenes ${ }^{[21]}$ chemisorbed on gold nanoparticles via alkylthiol linkers with a range of chain lengths have been reported. In these studies the effect of immobilisation on photochemical properties was of considerable interest both from molecule-surface and molecule-molecule interactions. Self-assembly of photoswitches on metallic (nanoparticulate) surfaces can result in direct electronic coupling of a nanoparticle core with a chromophore due to their proximity. ${ }^{[22,23]}$ This might result in complete inhibition of photoreactivity. Furthermore steric constraints can limit the conformational freedom of the molecular entities. For diarylethene-based photochromic switches, however, inhibition of photochemical activity because of steric interactions arising from packing in a monolayer is not expected to be significant especially considering that photochemistry is seen for this class of compound even in crystalline states. ${ }^{24]}$ The earliest study of diarylethenes self-assembled on a surface of gold nanoparticles employed pentamethylene alkyl chains to isolate the photoactive unit from the gold surface and showed full retention of the photochemistry observed in solution. ${ }^{[25]}$

This approach to preserving photochemistry by insulation from the surface does have a drawback in that some degree of orbital interaction with the surface is perceived as being essential to achieving resonant tunneling. Hence we deemed it essential to assess the effect of direct attachment of the dithienylethene photoswitches to metallic surfaces, both gold nanoparticles ${ }^{[26]}$ and electrodes ${ }^{[23]}$ using a range of conjugated aromatic spacers. For several spacer units the ring-opening process was retained. ${ }^{[23,26]}$ However ring closure showed a considerable dependence on the spacer group employed. The quantum yields for ring closing for meta- and para-

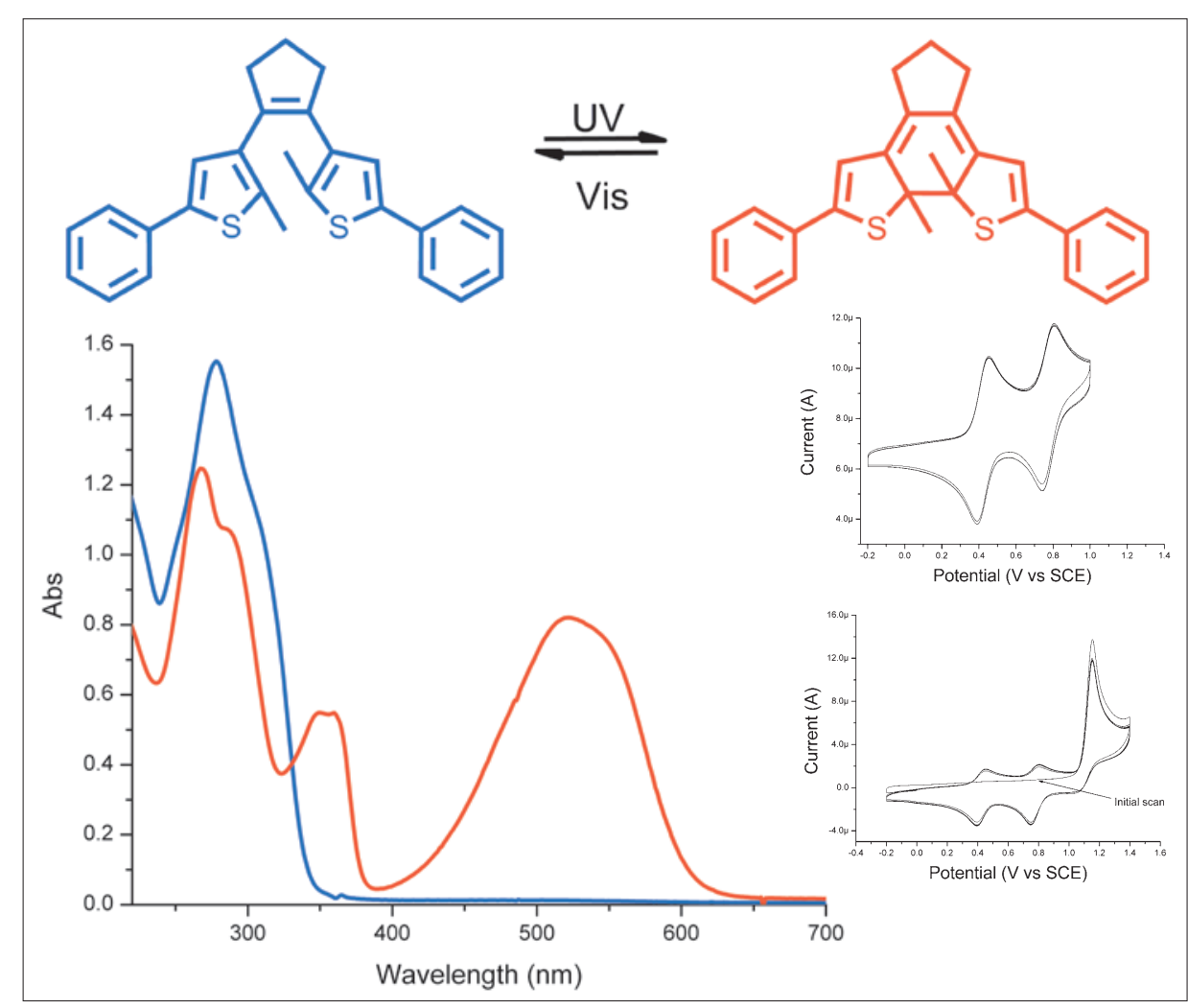

Fig. 1. UV/Vis absorption spectra and cyclic voltammetry of a dithienylethene photochromic switch in its ring opened and ring closed state. 
substituted phenyl units were the same indicating that the $\pi$-conjugation pathway has no effect on the electronic coupling of the chromophore with the Au surface. By contrast with a thienyl spacer group ring closing was essentially absent. ${ }^{[15,23,26]}$ The distance between the surface and the dithienylethenes is the same for all three systems and hence we can reasonably infer that the origin of differences in photochemical activity on the surface is due to perturbation of molecular orbital levels by the gold nanoparticles. ${ }^{[27]}$

\section{Light Switching of Conductance of Individual Molecules on Surfaces}

Achieving the goal of light-controlled molecular electronic devices is no simple feat. The first step is to observe phototriggered changes in conductance where a molecule bridges two electrodes. Furthermore this phenomenon needs to be demonstrated under conditions close to that which real molecular devices would operate, i.e. at ambient temperatures and self-assembled on nano-electrodes. The first challenges faced relate to discrimination of resonant tunnelling controlled by photoswitching from especially the stability of the molecule electrode interface and stochastic rearrangements of both surface and molecule. On a practical side placing individual molecules between electrodes is most easily achieved using scanning tunneling microscopy ${ }^{[28,29]}$ (STM) and the mechanically controlled break-junction ${ }^{[30]}$ (MCBJ). A further challenge is to avoid electrochemically induced switching, however by controlling the electrode potentials such voltage-induced switching can be avoided. ${ }^{[31]}$

With diarylethene photochromic switches (Fig. 2) rearrangement of double and single bonds upon photoswitching was expected to result in a significant change in conductance but without substantial change to their physical height when arranged vertically in a self-assembled monolayer. The first study of diarylethenes as components in molecular electronic devices employed the MCBJ technique in which two gold electrodes were bridged by a dithienylethene switch with anchoring to the surface achieved using thiol end groups. ${ }^{[15]}$ These early experiments revealed two key results. The first was that upon changing from a ring closed to a ring open form the conductance dropped by three orders of magnitude. Secondly the immobilisation of the switch between two electrodes completely prevented ring closing of the dithienylethene. Further studies using a monothiol functionalised dithienylethene in an STM confirmed these observations. ${ }^{[32]}$ These studies highlighted

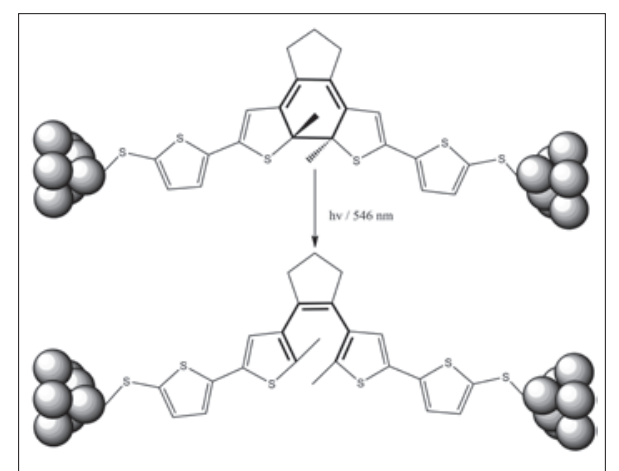

Fig. 2. Schematic representation of the switching from the closed to the open form using visible light for a dithienylethene bridging a mechanically controlled break junction.

a further challenge to molecular electronics; stochastic switching, which was voltage dependent and believed to be related to the electrochemical switching observed for these systems as well as the mobility of gold atoms in MCBJ devices. The absence of reversibility has been explored theoretically by several groups and although the initial view was that this is due to excited state quenching by gold, ${ }^{[33]}$ more recent analysis suggests it is more subtle a phenomenon where the interaction with gold serves to invert the relative ordering of molecular orbitals (i.e. lowering of a nonphotoactive excited state upon binding to gold). ${ }^{[27]}$ One approach to overcoming this

drawback is to reduce the efficiency of the contact between the gold surface and the dithienylethene using a methylene spacer group. The isolation of the switching unit with only a partly conjugated linker allowed for the retention of reversible photochromism. ${ }^{[34]}$ Although the differences between the two states is dramatically reduced taking this approach a switch in resistance from $526 \mathrm{M} \Omega$ in the open form and $4 \mathrm{M} \Omega$ in the closed form could be achieved. Replacing the gold electrodes with carbon nanotubes as demonstrated recently by the group of Guo and Nuckolls ${ }^{[35]}$ allows for further improvements in the approach by eliminating the problem of the mobility of gold atoms on the electrode surfaces.

Switching isolated individual dithienylethene switches between an open and a closed state with UV and visible light has been demonstrated recently. ${ }^{[36]}$ The individual molecules were isolated by dilution into self-assembled monolayers of n-dodecanethiol on Au(111) (Fig. 3). This approach simplified imaging the individual molecules. The state of the molecule (either ring opened or closed) was more readily observed due to the strong contrast with the surrounding alkyl thiols. The change in geometry e.g. height, observed for azobenzenes does not occur for dithienylethenes and hence the changes in tunnelling current observed reflect real changes in conductivity. A key advantage over the MCBJ

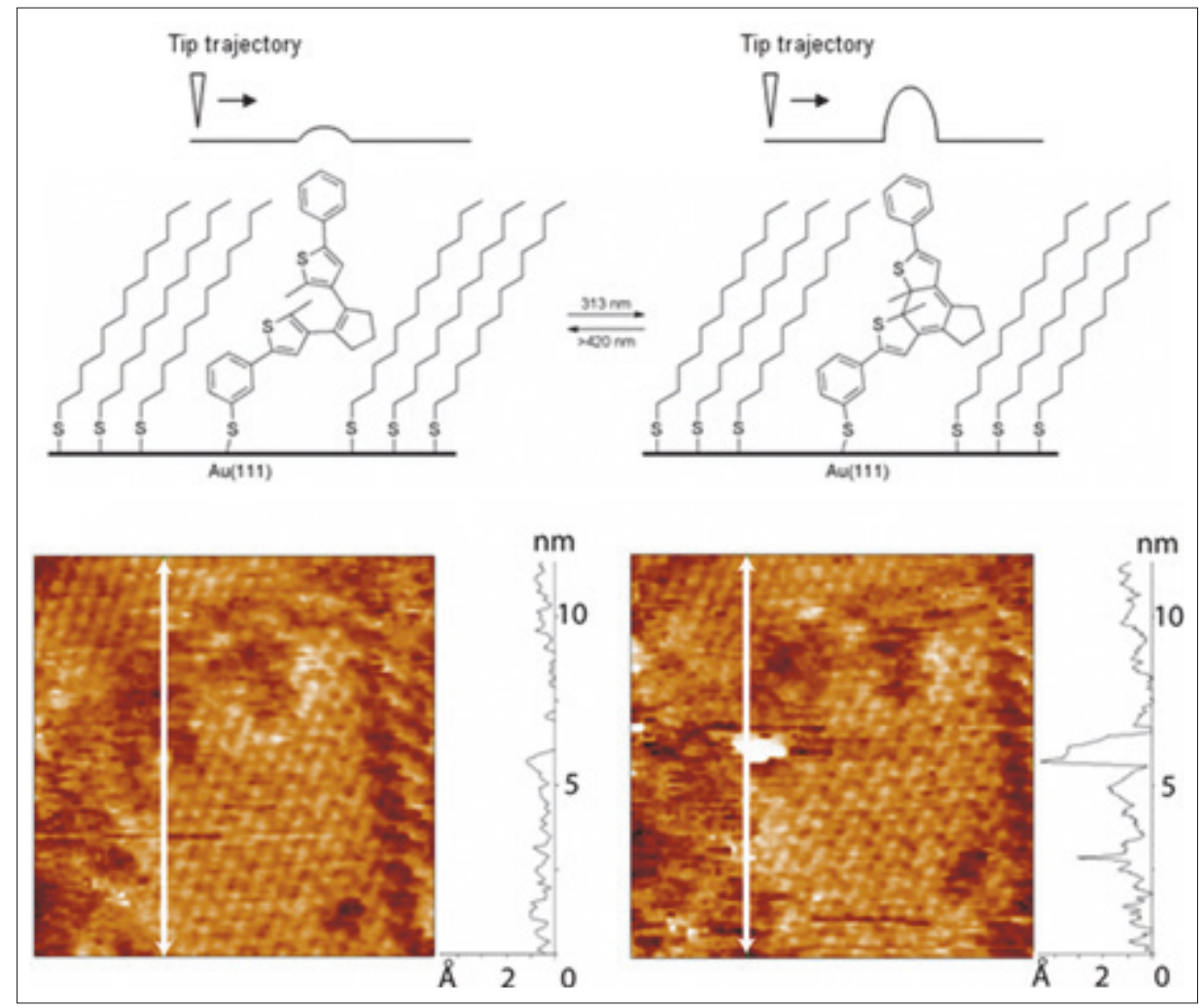

Fig. 3. (Upper) STM tip trajectory over individual open and closed dithienylethenes embedded in a dodecanethiol SAM on gold. (Lower) STM images of before (left) and after (right) switching with UV light. Reproduced with permission from ref. [36]. Copyright 2007 Wiley. 
A)

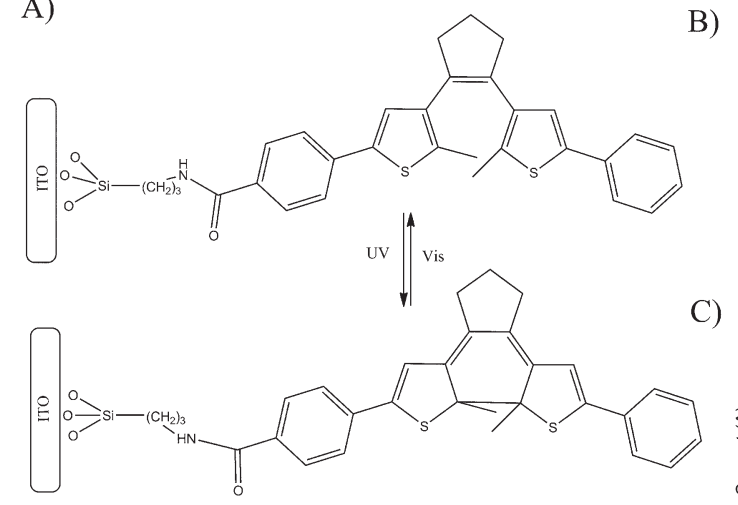

Fig. 4. A) Switching of a dithienylethene attached to an ITO electrode. B) Cyclic voltammetry of a ITO electrode coated with a monolayer of the open switch. Upon oxidation the dithienylethene switch undergoes ring closure quantitatively. C) before (a) and after (b) irradiation of a closed dithienylethene monolayer modified ITO electrode with visible light and (c) after electrochemical ring closing. Reproduced with permission from ref. [11]. Copyright 2006 RSC.

technique is the ability to perform statistically large numbers of experiments.

\section{Molecular Redox Switches}

The connection between photochemical and electrochemical switching is perhaps an under appreciated advantage in efforts to achieve multi-addressable molecular-based functional components for molecular devices. ${ }^{[37]}$ Electrochemistry allows entire monolayers of redox active molecular switches to be addressed at electron transfer limited rates and with high sensitivity. An example of this is shown in Fig. 2 and Fig. 4.[11] Cyclic voltammetry allows for changes in the energies of the HOMO and LUMO levels to be estimated and provides detailed insight as to whether it is the HOMO or LUMO orbitals or both change in energy upon switching. This information is key to interpreting the magnitude of changes in resonant tunnelling contributions in molecular electronic devices.

The simplest demonstration of switching of conductivity of molecules is perhaps achieved by electrochemical readout of a monolayer on an electrode surface. ${ }^{[11,23,38,39]}$ Recently we, and others, have employed electrochemistry as both a complimentary switching method to photoswitching as well as a read-out method for demonstrating the retention of photochemical properties of molecules after immobilisation of metal and semiconductor electrodes. ${ }^{[23,40,41]}$

Immobilising photochromic dithienylethene switches on surfaces as mono-

B)

)
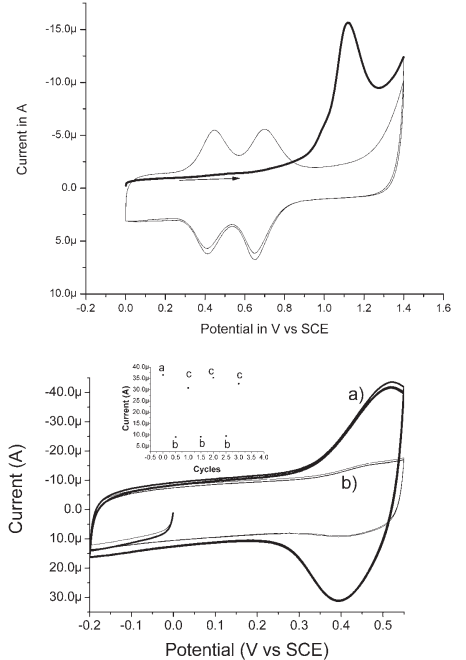

layers with high surface coverages allows for both the direct electronic interaction between the surface and the molecule ${ }^{[22]}$ (leading to quenching of photochemistry, changes to molecular orbital energies etc.), and steric effects, i.e. decreases in conformational freedom, changes to rates of intermolecular electron transfer, intermolecular interactions, and energy transfer to be observed. ${ }^{[12]}$ In solution dithienylethenes can be switched in both directions by UV and visible ir- radiation however redox-driven switching is typically only in one direction. ${ }^{[16]}$ Immobilisation on an ITO surface does not affect the photochemical properties of the dithienylethene shown in Fig. 4, however, in contrast to solution, on the surface it can be switched in both directions electrochemically. ${ }^{[11]}$ The difference in redox behaviour between in solution and on a surface is not due to changes in the properties of the switches or molecular orbital/ surface state interactions. Instead it occurs because the intermolecular electron transfer rates between the individual molecules is not diffusion limited as in solution since the molecules are held in proximity to each other on the surface.

\section{Photoswitching of Conductance in Organic Electronics}

A macroscopic switching device using diarylethene photochromes based polymers was reported recently by Irie and coworkers. ${ }^{[42]}$ A conductive dithienylethene-based polymer was sandwiched between ITO and metallic electrodes. Importantly this study demonstrated that different metal electrodes, e.g. Al, showed rectifying behaviour while, e.g., Au provided for symmetric IV characteristics. Stability was a key problem in the polymer-based devices, however, this could be overcome by using a non-photochromic polystyrene polymer doped with diarylethene molecules. ${ }^{[43]}$

Making working electronic devices based on molecules is a central goal of current nanoscience. A recent approach to photoswitching conductance was reported

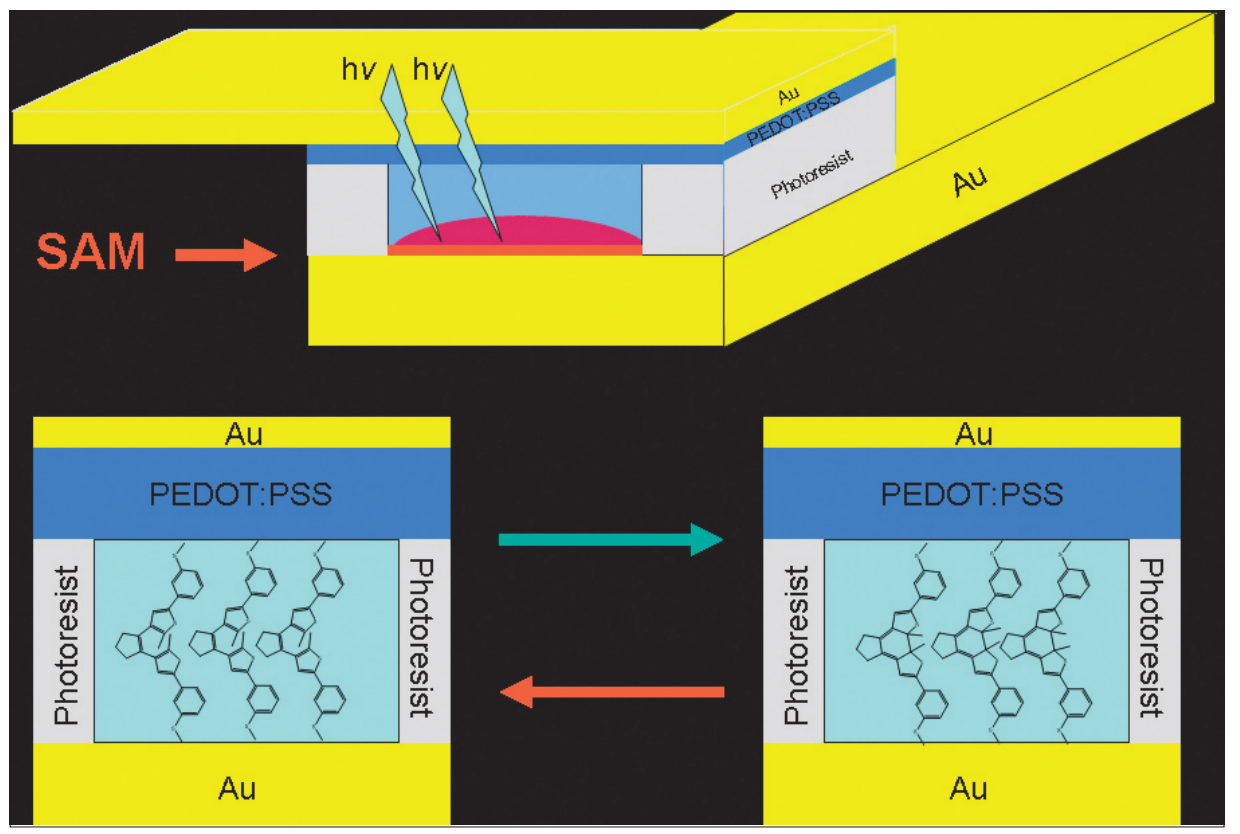

Fig. 5. Cross section diagram of the layout of a large-area molecular junction device where the diarylethene SAM is sandwiched between Au and PEDOT:PSS/Au. Adapted with permission from ref. [45]. Copyright 2008 Wiley. 
by Nuckolls and coworkers, ${ }^{[44]}$ in which a field effect transistor (FET) was constructed from a single-walled carbon nanotube (SWCNT) on which photochromic spiropyran switches were self-assembled and the SWCNT contacted by gold electrodes. UV and visible light irradiation could be used to switch between low and high conductance.

Recently a large array device that employed monolayers of diarylethene photochromic switches on a gold electrode covered with a PEDOT:PSS conducting polymer layer and a second layer of gold has demonstrated that light switching of conductance in an organic device is now a reality (Fig. 5). ${ }^{[45]}$ Importantly, the photochromic monolayer was coated with a conducting polymer layer to preclude short circuits through the photoactive SAM.[46] This key design innovation allowed for excellent reproducibility in the reversible photoswitching of conductance observed. Nevertheless challenges remain in particular in regard to device stability and in increasing the magnitude of the difference in conductivity achieved between the two photochromic states.

An alternative approach to self-assembled monolayers however is to use arrays of gold nanoparticles connected by dithienylethene chromophores. Micrometer-sized devices that use a nanoparticle network deposited between interdigitated nanogapped $\mathrm{Au}$ electrodes have been reported recently. ${ }^{[47]}$ Nanoparticles were coated with photoswitchable diarylethenes which served to interconnect individual nanoparticles. Fully reversible switching of conductance was achieved with sequential irradiation with UV and visible light. More recently this approach has been taken by our team together with colleagues to fabricate molecular devices in which light-controlled conductance switching could also be achieved. ${ }^{[32,48]}$ The devices were constructed using a two-dimensional lattice of gold nanoparticles in which adjoining particles were bridged by dithienylethene photoswitches. With this approach reversible isomerization of the diarylethenes at room-temperature resulted in conductance switching.

\section{Conclusions and Prospects}

Molecular electronics still faces considerable challenges before the field can make a substantial breakthrough from fundamental science to applications. This does not mean that substantial progress has not been made over the last two decades; far from it. The challenges in imaging individual molecules and interfacing them with bulk metal junctions have stimulated important developments in technology. It has also allowed us to appreciate the power of synthetic chemistry in preparing the molecular components in an increasingly made to order manner where the properties desired for a particular application are hard wired into the design of the molecule itself. The challenges presented to synthetic chemistry are to deliver improved molecular switches with faster response times, stability and reversibility over 100,000 s of operations. A further question to be addressed is the anchor employed to hold the molecules on the surface.

It remains to be seen how large a change in conductance can be achieved using individual molecules or in monolayers $^{[2]}$ and the dimensions the molecules must reach so that resonant tunnelling becomes the dominant parameter in controlling conductance; in this a collaborative multidisciplinary approach is paramount. Only once this has been established can we seriously consider more complex functions such as rectification. From the technical side, much more understanding of the phenomena which occur at the molecule/ electrode interface both upon attachment and during operation is necessary also.

What makes molecular and organic electronics, in our view, so exciting and hold so much promise for the future is that we have only begun to access the molecular tool box in the use of diarylethenes, azobenzenes, spiropyrans and stilbenes. Photo/redox active systems such as the overcrowded alkenes, ${ }^{[49]}$ and multiple switching systems based on oligothiophenes, ${ }^{[50]}$ could open much greater complexity in function with minimal increase in synthetic complexity. To date, molecules containing only a single switching unit have been employed, however it is expected that future efforts will be directed to larger molecular systems which allow for multiple switching units to be incorporated between the metal contacts of junctions. This approach although synthetically challenging allows both for increasing the junction gap size (thereby reducing direct tunnelling), amplifying the effect of changes in molecular structure and conductivity and in allowing for several conductivity states to be accessed (i.e. non-binary). A key challenge from a molecular design perspective is to achieve switching of multiple chromophores without loss of electronic communication between the units. Ultimately the true power of molecular electronics will not be in mimicking conventional solid state devices but in providing new operations through the use of combinations of molecular switching components.

Photo and redox switchable components present a bright future in organic and molecular electronics and, although considerable challenges remain to be overcome, the leap from demonstration of physical phenomena in molecular-based devices, such as the working redox device based on rotaxanes by Stoddart, Heath and coworkers $^{[5]}$ and more recent examples of light-driven solid-state molecular switching devices based on monolayers of photochromic diarylethene molecules, ${ }^{[45,46]}$ to application may be realised in the very near future.

\section{Acknowledgements}

This work was supported by NanoNed, the Zermike Institute (Depth Strategy), and the Netherlands Organization for Scientific Research (NWO-CW) through a VIDI grant (WRB).

Received: April 20, 2010

[1] E. A. Weiss, J. K. Kriebel, M. A. Rampi, G. M. Whitesides, Phil. Trans. Royal Soc. A. Math. Phys \& Eng. Sci. 2007, 365, 1509.

[2] a) E. A. Weiss, R. C. Chiechi, G. K. Kaufman, J. K. Kriebel, Z. F. Li, M. Duati, M. A. Rampi, G. M. Whitesides, J. Am. Chem. Soc. 2007, 129, 4336; b) E. Tran, M. Duati, V. Ferri, K. Mullen, M. Zharnikov, G. M. Whitesides, M. A. Rampi, Adv. Mater. 2006, 18, 1323.

[3] Y. Wada, Pure Appl. Chem. 1999, 71, 2055.

[4] A. Aviram, M. Ratner, Chem. Phys. Lett. 1974, 29, 277.

[5] J. E. Green, J. W. Choi, A. Boukai, Y. Bunimovich, E. Johnston-Halperin, E. DeIonno, Y. Luo, B. A. Sheriff, K. Xu, Y. S. Shin, H.-R. Tseng, J. F. Stoddart, J. R. Heath, Nature 2007, $445,414$.

[6] For a recent review of the field see: T. Kudernac, N. Katsonis, W. R. Browne, B. L. Feringa, J. Mater. Chem. 2009, 19, 7168.

[7] a) M. Irie, Chem. Rev. 2000, 100, 1685; b) B. L. Feringa, 'Molecular Switches', Wiley-VCH, Weinheim, Germany, 2001; c) H. Tian, S. J. Yang, Chem. Soc. Rev. 2004, 33, 85.

[8] a) H. Tian, Y. Feng, J. Mater. Chem. 2008, 18 , 1617 ; b) N. Katsonis, M. Lubomska, M. M. Pollard, B. L. Feringa, P. Rudolf, Prog. Surf. Sci. 2007, 82, 407.

[9] S. L. Gilat, S. H. Kawai, J.-M. Lehn, Chem. Eur J. 1995, 1, 275 .

[10] K. Uchida, M. Saito, A. Murakami, T. Kobayashi, S. Nakamura, M. Irie, Chem. Eur. J. 2005, 11, 534; b) M. Irie, 'Applied Photochromic Polymer Systems', Ed. C. B. McArdle, Blackie, Glasgow, 1991, pp. 174; c) T. Ikeda, O. Tsutsumi, Science 1995, 268, 1873; d) S. Shinkai, Pure Appl. Chem. 1987, 59, 425.

[11] J. Areephong, W. R. Browne, N. H. Katsonis, B. L. Feringa, Chem. Commun., 2006, 3930.

[12] W. R. Browne, Coord. Chem. Rev. 2008, 252, 2470.

[13] X.-Y. Zhu, Surf. Science Reports 2004, 56, 1

[14] T. Albrecht, A. Guckian, A. M. Kuznetsov, J. G. Vos, J. Ulstrup, J. Am. Chem. Soc. 2006, 128, 17132.

[15] D. Dulic, S. J. van der Molen, T. Kudernac, H. T. Jonkman, J. J. D. de Jong, T. N. Bowden, J. van Esch, B. L. Feringa, B. J. van Wees, Phys. Rev. Lett. 2003, 91, 207402

[16] a) W. R. Browne, J. J. D. de Jong, T. Kudernac, M. Walko, L. N. Lucas, K. Uchida, J. H. van Esch, B. L. Feringa, Chem. Eur. J. 2005, 11, 6414 and references therein; b) W. R. Browne, J. J. D. de Jong, T. Kudernac, M. Walko, L. N. Lucas, K. Uchida, J. H. van Esch, B. L. Feringa, Chem. Eur. J. 2005, 11, 6430 and references cited therein; c) G. Guirado, C. Coudret, M. Hliwa, J. P. Launay, J. Phys. Chem. B 2005, 109, 17445; d) B. Gorodetsky, H. D. Samachetty, R. 
L. Donkers, M. S. Workentin, N. R. Branda, Angew. Chem. Int. Ed. 2004, 43, 2812; e) A. Peter, N. R. Branda, J. Am. Chem. Soc. 2003 125, 3404; f) A. Peters, N. R. Branda, Chem. Commun. 2003, 4, 954.

[17] a) J. Hu, J. Zhang, F. Liu, K. Kittredge, J. K. Whitesell, M. A. Fox, J. Am. Chem. Soc. 2001 123, 1464. b) J. Zhang, J. K. Whitesell, M. A Fox, Chem. Mater. 2001, 13, 2323.

[18] A. Manna, P. L. Chen, H. Akiyama, T. X. Wei, K. Tamada, W. Knoll, Chem. Mater. 2003, 15, 20.

[19] K. Tamada, H. Akiyama, T. X. Wei, Langmuir 2002, 18, 5339.

[20] M. O. Wolf, M. A. Fox, Langmuir 1996, 12, 955.

[21] R. A. van Delden, M. K. J. ter Wiel, M. M. Pollard, J. Vicario, N. Koumura, B. L. Feringa, Nature 2005, 437, 1337.

[22] K. G. Thomas, P. V. Kamat, Acc. Chem. Res. 2003, 36, 888 .

[23] W. R. Browne, T. Kudernac, N. Katsonis, J. Areephong, J. Hjelm, B. L. Feringa, J. Phys. Chem. C 2008, 112, 1183 .

[24] a) S. Kobatake, S. Takami, H. Muto, T. Ishikawa M. Irie, Nature 2007, 446, 778; b) M. Irie, S Kobatake, M. Horichi, Science 2001, 291, 1769; c) K. Uchida, S. I. Sukata, Y. Matsuzawa, M. Akazawa, J. J. D. de Jong, N. Katsonis, Y. Kojima, S. Nakamura, J. Areephong, A. Meetsma, B. L. Feringa, Chem. Commun. 2008 326.

[25] K. Matsuda, M. Ikeda, M. Irie, Chem. Lett. 2004, 33, 456

[26] T. Kudernac, S. J. van der Molen, B. J. van Wees, B. L. Feringa, Chem. Commun. 2006 , 3597.

[27] a) K. Yoshizawa, T. Tada, A. Staykov, J. Am. Chem. Soc. 2008, 130, 9406; b) A. Staykov, K Yoshizawa, J. Phys. Chem. C, 2009, 113, 3826.

[28] a) P. Jiang, G. M. Morales, W. You, L. Yu, Angew. Chem. Int. Ed. 2004, 43, 4471; b) J. He,
Q. Fu, S. M. Lindsay, J. W. Ciszek, J. M. Tour, J. Am. Chem. Soc. 2006, 128, 14828; c) L. A. Bumm, J. J. Arnold, T. D. Dunbar, D. L. Allara, P. S. Weiss, J. Phys. Chem. B 1999, 103, 8122; d) B. Xu, N. J. Tao, Science 2003, 301, 1221.

[29] M. J. Comstock, N. Levy, A. Kirakosian, J. Cho, F. Lauterwasser, J. H. Harvey, D. A. Strubbe, J. M. J. Fréchet, D. Trauner, S. G. Louie, M. F. Crommie, Phys. Rev. Lett. 2007, 99, 038301.

[30] a) C. Kergueris, J.-P. Bourgoin, S. Palacin, D. Esteve, C. Urbina, M. Magoga, C. Joachim, Phys. Rev. B 1999, 59, 12505; b) M. Mayor, H. B. Weber, J. Reichert, M. Elbing, C. von Hänisch, d. Beckmann, M. Fisher, Angew. Chem. Int. Ed. 2003, 42, 5834; c) J. Reichert, R. Ochs, D. Beckmann, H. B. Weber, M. Mayor, H. von Lohneysen, Phys. Rev. Lett. 2002, 88, 176804.

[31] S. Yasuda, T. Nakamura, M. Matsumoto, H. Shigekawa, J. Am. Chem. Soc. 2003, 125, 16430 .

[32] S. J. van der Molen, H. van der Vegte, T. Kudernac, I. Amin, B. L. Feringa, B. J. van Wees, Nanotechnology 2006, 17, 310.

[33] a) J. Li, G. Speyer, O. F. Sankey, Phys. Rev. Lett. 2004, 93, 248302; b) M. Zhuang, M. Ernzerhof, Phys. Rev. B: Condens. Matter Mater. Phys. 2005, 72, 073104.

[34] J. He, F. Chen, P.A. Liddell, J. Andreasoon, S.D. Straight, D. Gust, T.A. Moore, A.L. Moore, J. Li, O.F. Sankey, S.M. Lindsay, Nanotechnology 2005, 16, 695.

[35] A. C. Whalley, M. L. Steigerwald, X. Guo, C. Nuckolls, J. Am. Chem. Soc. 2007, 129, 12590.

[36] N. Katsonis, T. Kudernac, M. Walko, S. J. van der Molen, B. J. van Wees, B. L. Feringa, $A d v$. Mater. 2006, 18, 1397.

[37] M. Pita, E. Katz, Electroanal. 2009, 21, 252

[38] a) I. Willner, A. Doron, E. Katz, J. Phys. Org. Chem. 1998, 11, 546; b) I. Willner, V. PradoYissar, E. Katz, K. T. Ranjit, J. Electroanal. Chem. 2001, 497, 172.
[39] E. Katz, B. Willner, I. Willner Biosensors \& Bioelect. 1997, 12, 703.

[40] R. Baron, A. Onopriyenko, E. Katz, O Lioubashevski, I. Willner, S. Wang, H. Tian, Chem. Commun. 2006, 2147.

[41] I. Willner, B. Basnar, B. Willner, Adv. Funct. Mater. 2007, 17, 702.

[42] T. Kawai, Y. Nakashima, T. Kunitake, M. Irie, Curr. Appl. Phys. 2005, 5, 139.

[43] E. Kim. M. Kim, K. Kim, Tetrahedron 2006, 62, 6814.

[44] X. Guo, L. Huang, S. O'Brien, P. Kim, C. Nuckolls, J. Am. Chem. Soc. 2005, 127, 15045.

[45] A. J. Kronemeijer, H. B. Akkerman, T. Kudernac, B. J. van Wees, B. L. Feringa, P. W. M. Blom, B. de Boer, Adv. Mater. 2008, 20, 1467.

[46] E. C. P. Smits, S. G. J. Mathijssen, P. A. van Hal, S. Setayesh, T. C. T. Geuns, K. A. H. A. Mutsaers, E. Cantatore, H. J. Wondergem, O. Werzer, R. Resel, M. Kemerink, S. Kirchmeyer, A. M. Muzafarov, S. A. Ponomarenko, B de Boer, P. W. M. Blom, D. M. de Leeuw, Nature 2008, 455, 956.

[47] M. Ikeda, N. Tanifuji, H. Yamaguchi, M. Irie, K. Matsuda, Chem. Commun. 2007, 1355.

[48] S. J. van der Molen, J. Liao, T. Kudernac, J. S. Agustsson, L. Bernard, M Calame, B. J. van Wees, B. L. Feringa, C Schoenenberger, Nano. Lett. 2009, 9, 76.

[49] W. R. Browne, M. M. Pollard, B. de Lange, A. Meetsma, B. L. Feringa, J. Am. Chem. Soc. 2006, 126, 12412.

[50] J. Areephong, J. H. Hurenkamp, M. T. W. Milder, A. Meetsma, J. L. Herek, W. R. Browne, B. L. Feringa, Org. Lett. 2009, 11, 721 . 